\title{
Automatización y control de un prototipo modular autosustentable para la producción de alimentos
}

\section{Automation and control of a self-sustaining modular prototype for food production}

CASTILLO-QUIROZ, Gregorio $\dagger^{*}$, CRUZ-GARRIDO, Arnulfo, ROJAS-BALBUENA, Dorian y LIMON-DIAZ, Miguel Ángel

Instituto Tecnológico Superior de Huauchinango, División de Ingeniería Mecatrónica y Maestría en Tecnologías de la Información

ID $1^{\text {er }}$ Autor: Gregorio, Castillo-Quiroz / ORC ID: 0000-0002-1904-4172, Researcher ID Thomson: H-9402-2018, arXiv Author ID: 0000-0002-1904-4172, CVU CONACYT ID: 162009

ID $1^{\text {er }}$ Coautor: Arnulfo, Cruz-Garrido / ORC ID: 0000-0001-9366-8525, Researcher ID Thomson: F-8400-2019, arXiv Author ID: 0000-0001-9366-8525, CVU CONACYT ID: 320172

ID $2^{\text {do }}$ Coautor: Dorian, Rojas-Balbuena / ORC ID: 0000-0002-4770-8669, Researcher ID Thomson: AAN-1530-2020, arXiv Author ID: 0000-0002-4770-8669, CVU CONACYT ID: 299726

ID $3^{\text {er }}$ Coautor: Miguel Angel, Limon-Diaz / ORC ID: 0000-0002-7578-7077, Researcher ID Thomson: T-6486-2017, arXiv Author ID: 0000-0002-7578-7077, CVU CONACYT ID: 349952

DOI: $10.35429 / J I E .2020 .12 .4 .42 .53$

Recibido Abril 20, 2020; Aceptado Junio 30, 2020

\section{Resumen}

La automatización de actividades productivas mediante soluciones tecnológicas es una apuesta clara para la disminución en costos de producción y un mayor control en los parámetros de calidad y seguridad. Es por eso que la tecnificación en el área de alimentos para acelerar la producción de alimentos vegetales y animales que reduzcan los costos y contrarresten la escasez. El presente artículo presenta el desarrollo de un prototipo modular vertical autosustentable para la producción de hortalizas y carne (aves de corral y peces) para la región de la Sierra Norte de Puebla, mediante el diseño e integración de un sistema de control automatizado respondiendo a la necesidad de proporcionar una alternativa en la producción de alimentos. El prototipo está compuesto por tres módulos: el primer módulo es para la crianza de peces, el segundo módulo es para la producción de hortalizas, el tercer módulo es para la crianza de aves de corral. De esta manera el proyecto pretende que a partir de la metodología puesta en marcha pueda ser implementada para la producción de alimentos en nuestra región.

Prototipo modular, Autosustentable, Alimentos

\begin{abstract}
Since 2009, Mexico faces a crisis due to scarcity and the consequent increase in prices of basic products. This situation is expected to continue, because the area available for cultivation, livestock and fishing is reaching its limit, while the population and its food needs are increasing. Today, strategies must be used to accelerate the production of plant and animal food that reduce costs and counter shortages. This article presents the development of a self-supporting vertical modular prototype for the production of vegetables and meat (poultry and fish) for the Sierra Norte region of Puebla, through the design and integration of an automated control system responding to the need to provide an alternative in food production. The prototype is made up of three modules: the first module is for raising fish, the second module is for vegetable production, the third module is for raising poultry. In this way, the project intends that, based on the methodology put in place, it can be implemented for food production in our region.
\end{abstract}

Modular prototype, Self-sustaining, Food

Citación: CASTILLO-QUIROZ, Gregorio, CRUZ-GARRIDO, Arnulfo, ROJAS-BALBUENA, Dorian y LIMON-DIAZ, Miguel Ángel. Automatización y control de un prototipo modular autosustentable para la producción de alimentos. Revista de Ingeniería Industrial. 2020. 4-12:42-53.

\footnotetext{
*Correspondencia al Autor (Correo Electrónico: gcquiroz1977@gmail.com)

$\dagger$ Investigador contribuyendo como primer autor.
} 


\section{Introducción}

La seguridad alimentaria es un concepto donde convergen aspectos de crecimiento económico, agropecuario y social. A partir de ellos, cada país trabaja para asegurar a sus habitantes el acceso a alimentos seguros, nutritivos y de acuerdo a sus necesidades. Diversos gobiernos y organismos multinacionales han hecho hincapié en la reducción del hambre y la desnutrición, dado que, según declaraciones de la Organización de las Naciones Unidas para la Agricultura y la Alimentación (FAO), el problema de la seguridad alimentaria radica en el acceso desigual a los alimentos, cambios climáticos, crisis económicas, conflictos y desplazamientos de población (Genesis, 2018).

Un informe presentado conjuntamente por la Unión Europea, la Organización de las Naciones Unidas para la Alimentación y la Agricultura (FAO) y el Programa Mundial de Alimentos (PMA), concluye que: en 2018, la cifra de 113 millones de personas que se enfrentan a crisis alimentarias, el número de personas en el mundo que padecen estas crisis alimentarias se ha mantenido por encima de los 100 millones en los últimos tres años, y el número de países afectados ha aumentado.

En este contexto, el Consejo Nacional de la Evaluación Política de Desarrollo Social (Coneval) en 2016 estimó la existencia de 53.4 millones de personas en situación de pobreza en el país, equivalente a 43.6 por ciento de la población total y de estas, 9.4 millones están en pobreza extrema, lo que en términos económicos limita el acceso a los alimentos necesarios para una adecuada alimentación y nutrición. Así también, debido a los constantes cambios climáticos surge la necesidad de cuidar la producción de alimentos desde su cultivo o crianza hasta el consumo con la finalidad de asegurar la producción y salud en nuestra población. Los métodos clásicos de producción de alimentos están cambiando constantemente, sobre todo con la incorporación de la tecnología de vanguardia, como resultado se generen sistemas de producción altamente rentables y eficientes.
Hoy en día los alimentos locales, sin pesticidas y cuya producción no genere emisiones ahora son posibles más allá de pequeños huertos urbanos. Pues como afirma el autor Lidia (2018), la producción a escala industrial responde al nombre de agricultura vertical. Las ventajas de la agricultura vertical son múltiples: reduce el espacio de producción, ahorra agua hasta en un $95 \%$, produce todo el año independientemente del clima; logra una mayor producción en menos tiempo, reduce el gasto en transporte, reduce las emisiones de $\mathrm{CO}_{2}$ y acerca la agricultura a las ciudades. El país de Holanda fue pionera con la primera granja vertical que operó comercialmente, la compañía Staay Food Group la instaló en Dronten. Pero a nivel mundial uno de los grandes referentes es Aerofarms, la compañía que patentó tecnología LED para que las plantas pudieran hacer la fotosíntesis, con la mayor eficiencia energética posible. Con este sistema, ya no hay límites para la producción alimentaria, ni la escasez de agua ni la climatología ni la falta de espacio. En España también se encuentran proyectos que van encaminados hacia la agricultura vertical. Un ejemplo es la empresa Achipámpanos, reinventa los huertos verticales con la idea de que cada uno en su casa pueda instalar su propio invernadero vertical.

Ahora en el contexto de la región de la Sierra Norte de Puebla, específicamente en Huauchinango tiene una producción importante de aves de corral, crianza de peces y producción de hortalizas, el propósito del presente proyecto titulado "Automatización y control de un prototipo modular autosustentable para la producción de alimentos", se fundamenta en el diseño e integración de un sistema de control automatizado respondiendo a la necesidad de proporcionar una alternativa en la producción de alimentos: hortalizas, aves de corral y peces.

El prototipo contempla una parte estructural que soporta tres módulos verticalmente. El primer módulo está destinado a la crianza de peces con un sistema de filtraje, recirculación, censado de $\mathrm{PH}$, un sistema de calentamiento y oxigenación del agua. El segundo módulo está destinado a la producción de hortalizas, con un sistema para el control de los nutrientes necesarios de los vegetales, y a su vez, los desechos de estos sirvan de alimento a las aves de corral. 
El tercer módulo contempla la crianza de aves de corral, contando con un sistema que concentra y trata los desechos, que sirven como nutrientes para las hortalizas, de igual manera este módulo cuenta con un sistema de calefacción, un sistema de oxigenación, y un sistema de extracción de gas amoniaco, para evitar enfermedades.

El proyecto está enfocado para lograr un alto rendimiento del prototipo modular para la producción de hortalizas y carne (aves de corral y peces), e incorpora tecnología para poder controlar su desarrollo. De esta manera el proyecto pretende que a partir de la metodología puesta en marcha pueda ser implementada en la región de la Sierra Norte del Estado de Puebla en favor de la seguridad alimentaria.

El desarrollo de este trabajo de investigación está dividido en las secciones que a continuación se describen:

\section{Metodología.}

Se describen los pasos que se siguieron para el desarrollo del proyecto.

\section{Resultados.}

En esta sección se analizan los resultados obtenidos para determinar si se ha logrado el objetivo.

\section{Agradecimientos.}

Se agradece a las personas e instituciones que permitieron el desarrollo de esta investigación.

\section{Conclusiones.}

Se habla de los objetivos logrados satisfactoriamente y de cómo contribuye este trabajo en la producción de alimentos.

\section{Metodología}

El prototipo modular vertical autosustentable se divide en tres partes: el primer módulo es para la crianza de peces, el segundo módulo es para la producción de hortalizas, el tercer módulo es para la crianza de aves de corral, como se muestra en la Figura 1.

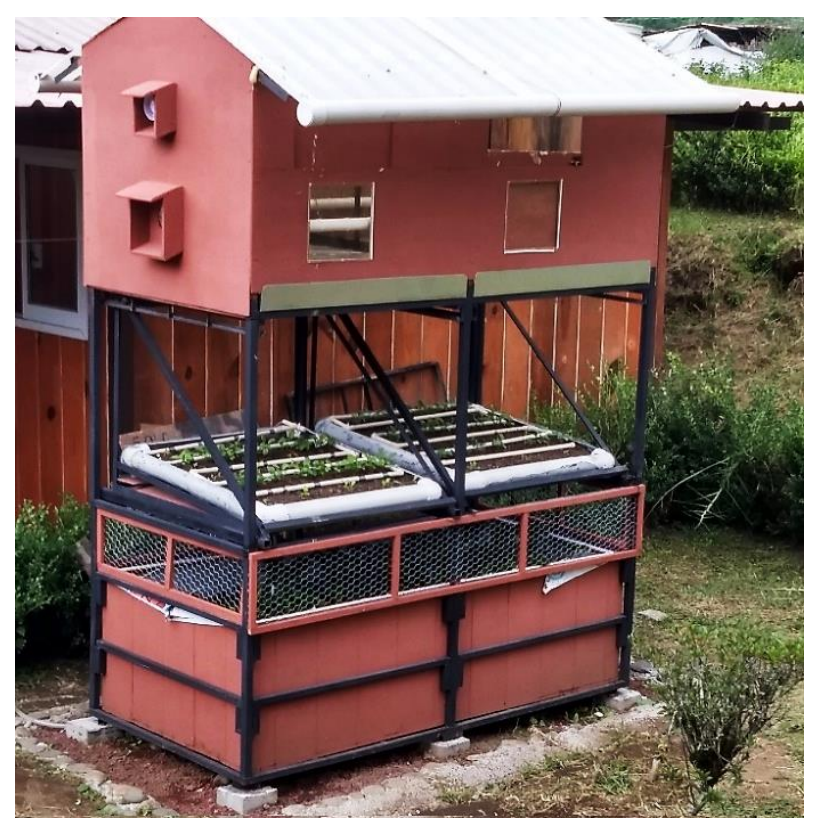

Figura 1 Prototipo modular autosustentable Fuente: Elaboración propia

El prototipo modular implementado busca la necesidad de garantizar el suministro continuo de alimentos e impactar de manera considerable en la economía familiar tanto en zonas rurales y urbanas al producir estos insumos de manera más eficiente y optimiza recursos mediante procesos automatizados, permitiendo generar dichos insumos incluso fuera de temporada. Se implementó en 3 niveles, organizado de manera vertical, considerando factores críticos para su fácil implementación y reducción de costos. En este enfoque, la estructura se basa en un perfil tubular cuadrado (PTR) y perfil angular, con diseño modular para que la producción y ensamble sean económicos y de sencillo acceso. Por consiguiente, un sistema básico de esta naturaleza tiene un costo aproximado de $40 \mathrm{mil}$ pesos.

\section{Materiales}

El prototipo modular consta con tres niveles, cada uno de ellos dedicado a la producción de cada una de dichas especies. Para la automatización y control del prototipo modular se precisó de las necesidades del proyecto, optando por la siguiente lista con sus respectivas especificaciones que se presenta en la Tabla 1: 


\begin{tabular}{|c|c|c|}
\hline Elementos & Especificaciones & Cantidad \\
\hline $\begin{array}{l}\text { Bomba de agua } \\
\text { de medio HP }\end{array}$ & $\begin{array}{l}\text { De medio HP. Además, } \\
\text { que funcione con un } \\
\text { voltaje de } 110 \text { VCA a } \\
60 \mathrm{~Hz} \text { monofásico. }\end{array}$ & 1 \\
\hline $\begin{array}{l}\text { Foco } \\
\text { incandescente }\end{array}$ & $\begin{array}{l}\text { De } 60 \text { watts, que } \\
\text { funcione con } 110 \mathrm{VCA} \\
\text { a } 60 \mathrm{~Hz}\end{array}$ & 3 \\
\hline Extractor & $\begin{array}{l}\text { Alimentación con } 110 \\
\text { VCA a } 60 \mathrm{~Hz} \text {. }\end{array}$ & 1 \\
\hline Ventilador & $\begin{array}{lrr}\text { Alimentación a } & 110 \\
\text { VCA a } 60 & \mathrm{~Hz} \\
\text { monofásico. } & & \\
\end{array}$ & 1 \\
\hline $\begin{array}{l}\text { Sensor de } \\
\text { temperatura } \\
\text { pt100 }\end{array}$ & 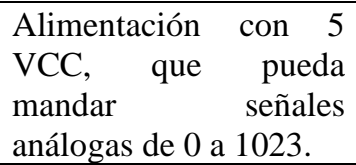 & 1 \\
\hline $\begin{array}{l}\text { Sensor de } \\
\text { humedad yl-19 }\end{array}$ & $\begin{array}{l}\text { Alimentación con } 5 \\
\text { VCC y mandar señales } \\
\text { análogas de } 0 \text { a } 1023\end{array}$ & 2 \\
\hline Sensor de nivel & $\begin{array}{lr}\text { Alimentación con } 5 \\
\text { VCC, tener la capacidad } \\
\text { de pasar de NA }- \text { NC y } \\
\text { estar compuesto } \\
\text { enteramente } \\
\text { material de tipo } \\
\text { polímero }\end{array}$ & 4 \\
\hline Sensor de PH & $\begin{array}{l}\text { Alimentación con } 5 \\
\text { VCC, mandar señales } \\
\text { análogas de } 0 \text { a } 1023 \text { y } \\
\text { que sea resistente al } \\
\text { agua }\end{array}$ & 1 \\
\hline $\begin{array}{l}\text { Conductor } \\
\text { eléctrico } \\
\text { calibre } 10\end{array}$ & Cable de 2 polos & $6 \mathrm{~m}$ \\
\hline $\begin{array}{l}\text { Conductor } \\
\text { eléctrico }\end{array}$ & $\begin{array}{l}\text { Cable a base de cobre, } \\
\text { calibre } 14\end{array}$ & $30 \mathrm{~m}$ \\
\hline $\begin{array}{l}\text { Conductor para } \\
\text { componentes } \\
\text { electrónicos } \\
\end{array}$ & $\begin{array}{l}\text { Cable UTP de un solo } \\
\text { hilo }\end{array}$ & 30 \\
\hline Soquet & Deberá ser a 120 VCA & 6 \\
\hline Clemas & 3 amperes cada uno & 10 Pzas. \\
\hline $\begin{array}{l}\text { Controlador } \\
\text { DAQ USB- } \\
6008\end{array}$ & $\begin{array}{lr}\begin{array}{l}\text { Sujeto } \\
\text { especificaciones } \\
\text { proveedor }\end{array} & \text { del } \\
\end{array}$ & 1 \\
\hline Arduino UNO & $\begin{array}{lr}\begin{array}{l}\text { Sujeto } \\
\text { especificaciones } \\
\text { proveedor }\end{array} & \text { del } \\
\end{array}$ & 1 \\
\hline Laptop & $\begin{array}{lr}\begin{array}{lr}\text { Sujeto } \\
\text { especificaciones } \\
\text { proveedor }\end{array} & \text { del } \\
\end{array}$ & 1 \\
\hline
\end{tabular}

Tabla 1 Especificaciones de los materiales Fuente: Elaboración propia

\section{Instalación de sistema de recirculación del agua}

El diseño del sistema de circulación del agua, se empleó el software de diseño de sistemas hidráulicos FluidSIM de Festo.
Cabe aclarar que algunos componentes al ser muy básicos sus símbolos, se colocaron elementos para simbolizar el tipo de proceso que se realiza en cada estación, obteniendo el diseño como se observa en Figura 2.

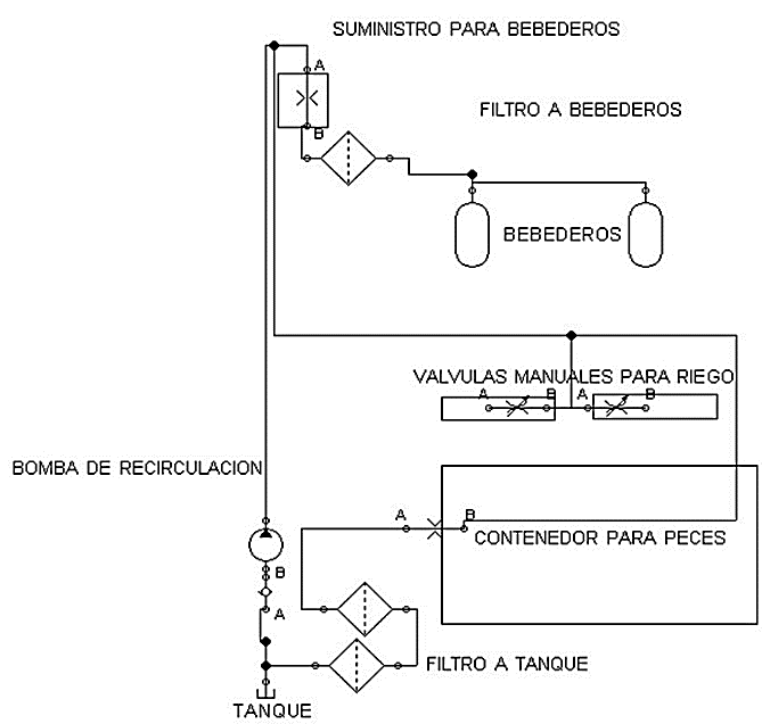

Figura 2 Diseño de sistema de recirculación del agua Fuente: Elaboración propia

Para la selección de la bomba se basó en la NORMA Oficial Mexicana NOM-004ENER-2014 para bombeo de agua limpia de uso doméstico, en potencias de $0.180 \mathrm{~kW}$ hasta $0.750 \mathrm{~kW}$. Se instaló una bomba de $1 / 2$ " hp, ubicándolo dentro del cuarto de control, para reducir los daños por estrés que el ruido de la bomba ocasiona al entrar en estado activo y pueda tener repercusiones tanto en los peces y en las aves.

Para suministrar el agua al prototipo se conectó en la salida de la bomba una manguera de $1 / 2$ " hasta la entrada del contenedor del agua para los bebederos en donde se colocó una $t$ de CPVC de $1 / 2$ " para dividir el caudal entre el contenedor y las tuberías empleadas para el riego de las hortalizas, por último el resto del agua en circulación caerá al estanque de los peces por medio de una tubería conectada en una división que se le hizo a la tubería del riego de las hortalizas como un método de oxigenación. Parte del agua que no se almacena en el contenedor de los bebederos es empleada para el riego de las hortalizas, por el método de goteo. El sistema de siembra permite que el agua empleado para regar las hortalizas se filtre y descienda al contenedor de peces favoreciendo a la oxigenación del agua en favor de los peces. 


\section{Diseño, maquinado e integración del circuito de opto-acoplado}

El diseño de la placa se realizó en el software PCB de PROTEUS, es para el control de actuadores a $127 \mathrm{v}$ de corriente alterna con una señal de la DAQ de 5 VCC.

Se optó por diseñar una sola placa que contenga a los circuitos de control de todos los actuadores a excepción de la bomba, siendo un total de 5 actuadores en la placa, con esto se optimiza el espacio dentro del cajón de mando brindando mejor estética y seguridad al disminuir la cantidad de cable que se empleó para la alimentación eléctrica, véase la Figura 3.

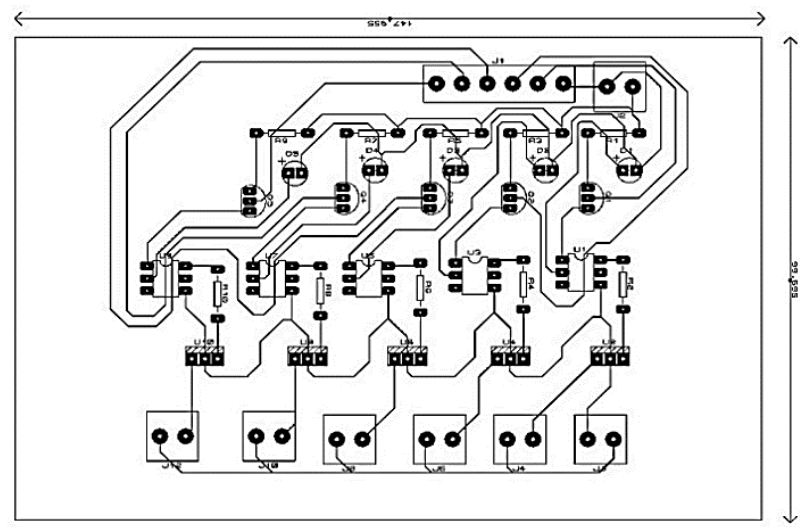

Figura 3 Diseño de placa general simulando todos los componentes

Fuente: Elaboración propia

Para el control de la bomba se diseñó otra placa (Figura 4) con características que permitieran controlar un potencial de mayor amperaje, debido al mayor consumo de la bomba, con esto se evita que por el consumo de potencial de la bomba se pueda producir sobrecalentamiento o corto circuito a los circuitos de control de los demás actuadores.

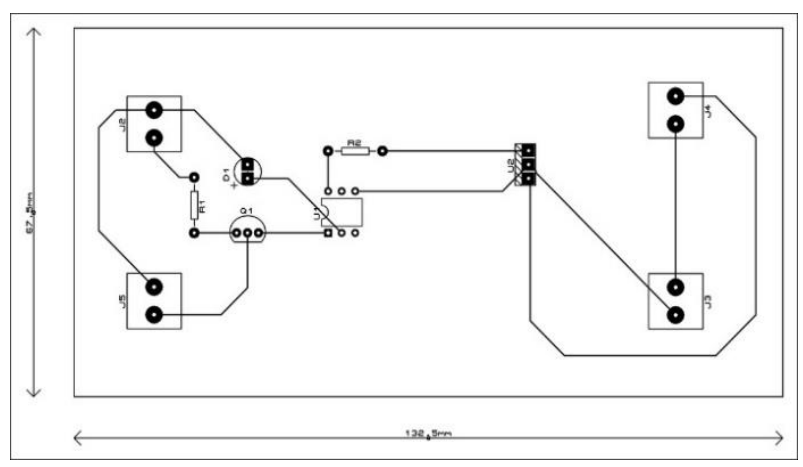

Figura 4 Diseño de placa para la bomba simulando componentes reales

Fuente: Elaboración propia
Una vez que los diseños estaban concluidos se obtuvieron los códigos para empezar con el maquinado de las placas. En el caso de la placa general se soldaron componentes para cinco circuitos de opto acoplado donde se conectarán el extractor, el ventilador, las lámparas de crecimiento, los focos incandescentes, véase Figura 5.

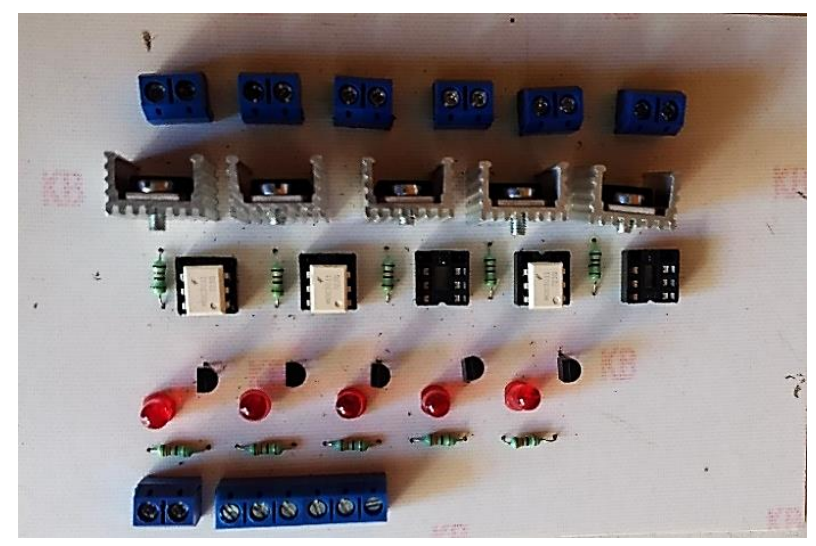

Figura 5 Placa general integrada

Fuente: Elaboración propia

\section{Cálculo de corriente total requerida}

Todos los componentes eléctricos que se adquirieron traen las especificaciones del consumo energético en potencia o sea en watts, para calcular la corriente necesaria se obtuvo que aplicar la ley de Watt.

Para calcular las protecciones eléctricas se debe hacer la suma total de todas las cargas en watts que consumen los componentes, esto se divide entre el voltaje con el que se cuenta o se quiere trabajar y el resultado es la corriente expresado en amperes que será multiplicado por el factor establecido en la NOM-101-SEDE2012, para instalaciones eléctricas Capítulo 2 Apartado 215 alimentadores y Capitulo 2 Apartado 240 protección contra sobrecalentamiento que es 0.25 , una vez obtenido el resultado y no hay protección exacta se debe elegir uno con el valor superior más próximo, en nuestro caso el voltaje proveniente de la toma corriente al cual se conectó la alimentación es de $110 \mathrm{VCA}$, realizando las operaciones mencionadas se obtuvo que la corriente $\mathrm{i}_{n}$ es de $25.2 \mathrm{~A}$. 


\section{Cálculo e instalación de conductores eléctricos}

Tomando en cuenta datos del consumo requerido por los actuadores, se compararon con respecto a valores determinados por la norma. Los requisitos establecidos en este proyecto tienen el propósito de garantizar la seguridad de las personas, animales y bienes contra los riesgos que puedan resultar de la utilización de las instalaciones eléctricas.

Para la alimentación general del prototipo se empleó cable calibre 12 AWG, debido a que es el que más idóneo por todos los actuadores que posee el sistema. El cableado para la alimentación de las lámparas de crecimiento y de alimentación y adquisición de datos para los sensores, se ruteó sobre la parte trasera de la estructura, dentro de unas canaletas hasta llegar a la caja de registro. De la caja de registro se trasladan los conductores introduciéndolos en manguera según la norma para instalaciones eléctricas.

Los cables provenientes de la caja denominada de registro son seleccionadas dependiendo del voltaje que trasladan las de potencia son recibidas por unas clemas que se encuentran dentro del cajón de mando. Posteriormente el cable de cada clema es conectado a un pin determinado en el circuito de potencia. Los cables señal para la adquisición de datos se conectaron a una Protoboard en el que a cada sensor tiene un circuito control, los módulos de linealizado de los sensores de humedad YL-19 igualmente fueron colocados en la Protoboard. Las salidas de los transistores se conectaron a los pines específicos de la DAQ determinados en el software de control, ver Figura 6, de esta manera el software podrá empezar a recibir los datos de los sensores, los comparará a los valores establecidos y ejecutará las acciones establecidas en el programa. Con esto la DAQ enviará una señal a un pin del circuito de potencia con lo cual se accionará el actuador necesario para regular las variables.

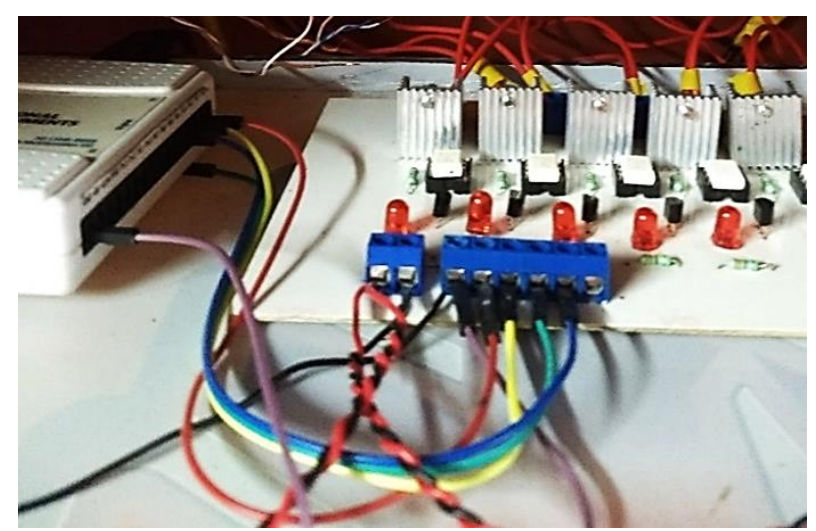

Figura 6 Conexión a DAQ

Fuente: Elaboración propia

\section{Tecnologías de control y propuesta de automatización}

El análisis e interpretación de las variables (temperatura y humedad relativa) permiten tener un referente de diseño, ensamble, revestimiento y colocación física del prototipo. También sirve para dimensionar y seleccionar el tipo de control a utilizar, los sensores y actuadores, así como, para la selección de valores de ajuste en los algoritmos de control y diseño de la interfaz de monitoreo, graficación y control de los tres módulos y sus alarmas de proceso. El sistema de control está basado en el controlador DAQ NI USB-6008, cuenta con 8 puertos de entrada que serven de entradas análogas o digitales para realizar la adquisición de datos de los sensores.

Los puntos de datos muestreados y establecidos forman parte del sistema de control y monitoreo. Proporcionan valores y una marca temporal, lo cual permite el monitoreo y el control, así como la generación de alarmas que permite la supervisión y la pronta atención en caso de haber falla. La construcción de una interfaz de usuario amigable y fácil de usar se empleó el entorno de desarrollo gráfico de LabVIEW 2019 aprovechando su compatibilidad con el controlador utilizado y recomendada para sistemas de pruebas de control y diseño, simulado o real y embebido. 


\section{Desarrollo del panel de control y visualización del nivel para la crianza de aves de corral}

En este módulo se realizó el sistema de adquisición de datos para sensar temperatura y humedad relativa para controlar el sistema de calefacción, oxigenación, y extracción de gas amoniaco, que tiende a enfermar de vías respiratorias a las aves. Además, un sistema de monitoreo del nivel de agua en el contenedor de agua para los bebederos.

Teniendo como base referente los siguientes datos mostrados en la Tabla 2, proporcionados por los manuales de avicultura.

\begin{tabular}{|r|r|}
\hline \multicolumn{1}{|c|}{ Edad en días } & Temperatura \\
\hline $1-7$ & $28-32{ }^{\circ} \mathrm{C}$ \\
\hline $8-14$ & $26-28^{\circ} \mathrm{C}$ \\
\hline $15-21$ & $24-26^{\circ} \mathrm{C}$ \\
\hline $22-28$ & $22-25^{\circ} \mathrm{C}$ \\
\hline $29-35$ & $20-22^{\circ} \mathrm{C}$ \\
\hline 36 al sacrificio & $20-22^{\circ} \mathrm{C}$ \\
\hline
\end{tabular}

Tabla 2 Guía de avicultura

Fuente: https://avicultura.info/medicion-de-parametrosproductivos-en-avicultural

Para el monitoreo y control de las variables en el galpón de las aves se diseñó un algoritmo en el que se empleó el sensor de humedad y temperatura DHT-11, un sensor de tipo switch, un extractor y un ventilador, y en un circuito separado una fotocelda y 2 focos incandescentes, un termopar tipo j, un pirómetro y una resistencia para generar calor. Una vez que la DAQ recibe los datos del sensor los compara, con los valores establecidos como ideales y en caso de que el valor de la temperatura sea mayor o igual a $30^{\circ} \mathrm{C}$, ejecutará una acción que deberá activar al extractor, así deberá empezarse a regular la temperatura. En caso del ventilador de oxigenación estará encendiéndose 15 minutos y apagándose 15 minutos para suministrar aire al interior de nave.

En la interfaz de usuario se hace la visualización del estado de los niveles de agua que intervienen en el suministro a los bebederos. En donde los indicadores de cada contenedor representan un sensor en la estructura real.
Después de hacer la instalación de los sensores y actuadores se diseñó el sistema de adquisición de datos para el sensor DHT11 ubicado en la nave de las aves, con el algoritmo programado para el sensor DHT-11 se obtiene la visualización de la humedad y la temperatura dentro del galpón por medio de indicadores analógicos. El estado del extractor y del ventilador se mostrará en el panel de visualización, ver Figura 7.

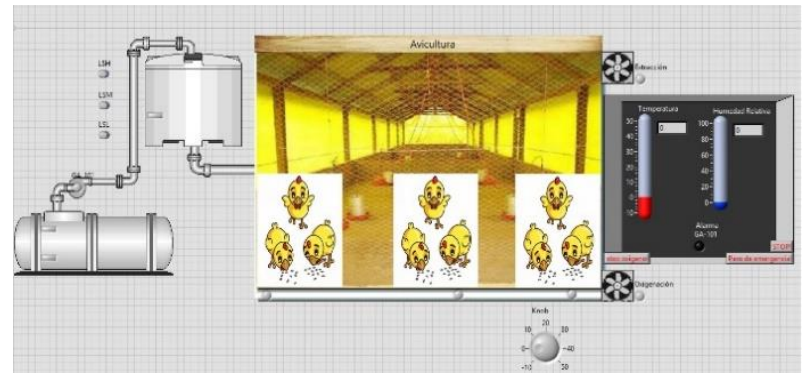

Figura 7 Panel de visualización del nivel para la crianza de aves de corral

Fuente: Elaboración propia

\section{Desarrollo del panel de control y visualización del nivel para el cultivo de hortalizas}

Partiendo de los datos proporcionados por la FAO mostrados en la Tabla 3 sobre la temperatura, humedad relativa y las condiciones de almacenamiento óptimo para las principales especies de frutas y verduras. Además, se consideró las condiciones climatológicas del Estado de Puebla, se eligieron las especies de hortalizas a rotar en la producción.

\begin{tabular}{|l|r|r|r|}
\hline \multicolumn{1}{|c}{ Especie } & \multicolumn{1}{c}{$\begin{array}{c}\text { Temperatura } \\
\left({ }^{\circ} \mathbf{C}\right)\end{array}$} & \multicolumn{1}{c|}{$\begin{array}{c}\text { Humedad } \\
\text { relativa } \\
(\%)\end{array}$} & $\begin{array}{c}\text { Tiempo de } \\
\text { almacenamiento } \\
\text { (días) }\end{array}$ \\
\hline Brócoli & 0 & $95-100$ & $14-21$ \\
\hline $\begin{array}{l}\text { Cebolla } \\
\text { bulbo }\end{array}$ & 0 & $65-70$ & $21-28$ \\
\hline $\begin{array}{l}\text { Cebolla de } \\
\text { verdeo }\end{array}$ & 0 & $95-100$ & $14-21$ \\
\hline Cebollino & 0 & $95-100$ & $10-14$ \\
\hline Espinaca & 0 & $95-100$ & $14-21$ \\
\hline Lechuga & $0-2$ & $98-100$ & $14-60$ \\
\hline Perejil & 0 & $95-100$ & $21-35$ \\
\hline Pimiento & $7-13$ & $90-95$ & $60-90$ \\
\hline $\begin{array}{l}\text { Repollo de } \\
\text { Bruselas }\end{array}$ & 0 & $95-100$ & $14-21$ \\
\hline $\begin{array}{l}\text { Repollo } \\
\text { chino }\end{array}$ & 0 & $95-100$ & $21-28$ \\
\hline $\begin{array}{l}\text { Tomate verde } \\
\text { maduro }\end{array}$ & $12.5-15$ & $90-95$ & 14 \\
\hline $\begin{array}{l}\text { Tomate rojo } \\
\text { maduro }\end{array}$ & $8-10$ & $90-95$ & $210-270$ \\
\hline $\begin{array}{l}\text { Tomate de } \\
\text { árbol }\end{array}$ & $3-4$ & $85-90$ & \\
\hline $\begin{array}{l}\text { Zanahoria } \\
\text { c/hoja }\end{array}$ & 0 & $95-100$ & \\
\hline $\begin{array}{l}\text { Zanahoria } \\
\text { s/hoja }\end{array}$ & 0 & $98-100$ & \\
\hline
\end{tabular}

Tabla 3 Condiciones de almacenamiento óptimo para las principales especies de hortalizas

Fuente: http://www.fao.org/3/y4893s/y4893s06.htm 
En la tabla anterior, se observa una constante de humedad relativa alta, que va del $85 \%$ al $100 \%$, se decidió colocar un sensor de humedad relativa en tierra, lo que determina la variable medida con respecto al valor de ajuste del $100 \%$ donde para la bomba de riego se le colocó una histéresis que va entre $80 \%$ y $90 \%$ según la hortaliza a producir, basado en los parámetros referentes y la humedad relativa censada, con el fin de activar la bomba y/o los aspersores.

Empleando un sensor de humedad en suelo YL-19 en cada cajón del sistema hidropónico para monitorear la humedad de la tierra, se diseñó el sistema de adquisición de datos para los sensores y el panel de visualización al usuario. Para hacer más fácil la interpretación de los datos de la DAQ únicamente será por medio de indicadores como se representa el estado de la humedad, en cada cajón se cuenta con tres indicadores a los cuales se le dio la nomenclatura de $\mathrm{S}=$ seca, $\mathrm{H}=$ húmeda y $\mathrm{MH}=$ muy húmeda. Los valores de cada nomenclatura están entre, $0.01 \mathrm{~V}-1 \mathrm{~V}=\mathrm{a} \mathrm{MH}$, de $1.2 \mathrm{~V}$ a $2.8 \mathrm{~V}=\mathrm{H}$ y de $3.1 \mathrm{~V}-3.5 \mathrm{~V}=\mathrm{S}$.

Para tener un buen desarrollo se instalaron lámparas de crecimiento cuya función es brindar luminosidad cuando exista escasez de luz solar, por lo que fue necesario desarrollar un sistema de adquisición de datos para trabajar con un sensor de luminosidad (LDR). En el panel de visualización se muestra el estado de la humedad por medio de indicador variable cuyo valor va de $0-5 \mathrm{~V}$ representando mayor humedad cuando exista menor voltaje respectivamente.

El código nos indica un comparador de valores de voltaje de $0 \mathrm{~V}-4 \mathrm{~V}$ cuyo valor se puede ver representado en los indicadores del panel de visualización (Figura 8) y en caso de que el valor se menor a 0 se envía la señal que debe activar a las lámparas y en cuanto se activen las lámparas de la estructura el indicador en el panel de visualización cambiará de igual manera a estado activo.

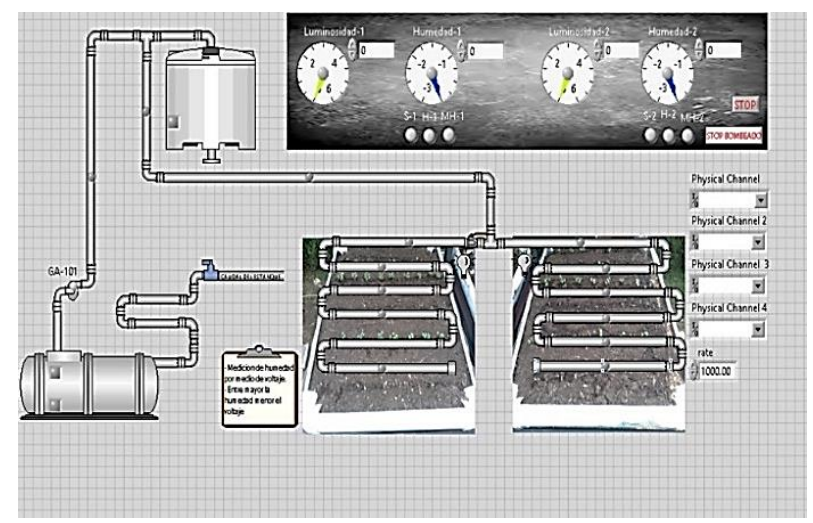

Figura 8 Panel de visualización del nivel para el cultivo de hortalizas

Fuente: Elaboración propia

\section{Desarrollo del panel de control y visualización del nivel para la crianza de peces}

El control se diseñó para mantener oxígeno suficiente controlado y cumplir con el ciclo de carbono para el mejor rendimiento y desarrollo de las especies, cabe mencionar que es necesario censar el pH del agua para el control de los sistemas de filtrado y retroalimentación, todo esto con un sistema de adquisición de datos y control automático.

En este caso se monitorea el nivel de $\mathrm{pH}$ del agua con el sensor E201-BNC colocado en el estanque, este sensor arroja la señal con voltaje, que con una ecuación se tratada para indicar el $\mathrm{pH}$ presente, al obtener el valor del $\mathrm{pH}$ se clasifica el valor de 9-14 unidades el $\mathrm{pH}$ es considerado alto por lo tanto la DAQ manda una señal al panel de visualización indicando una alarma, de 5 -9 el PH es considerado aceptable y cualquier valor menor a 5 será considerada baja por lo tanto también deberá activarse la alarma.

El sistema de almacenamiento y recirculación del agua es monitoreado mediante sensores todo o nada, se colocaron 2 sensores en el contendor enterrado, la función de estos sensores es activar y desactivar la bomba.

El estado de $\mathrm{pH}$ medido es visualizado por medio de indicadores de tipo booleano para expresar el rango e indicadores variables para expresar el valor en el panel de visualización, véase Figura 9. Además, en el panel se pueden ver indicadores del estado de los sensores de nivel en tanque bajo y tanque alto, así como indicadores boléanos para las alertas en caso de error en el almacenamiento del agua sea en tanque alto o tanque bajo. 


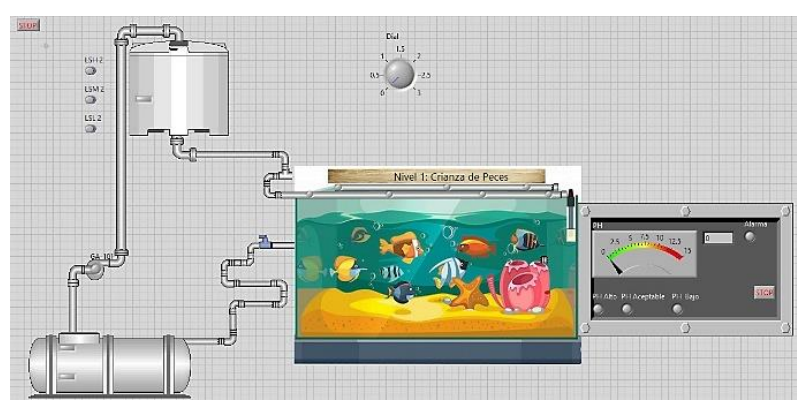

Figura 9 Panel de visualización del nivel para la crianza de peces

Fuente: Elaboración propia

\section{Resultados}

Una vez que los tres niveles de la estructura del prototipo se ensamblaron y adecuaron con los elementos necesarios, colocando desde un sensor, un actuador hasta desarrollando un software subdividido enfocado en controlar un nivel en específico, se implementó un sistema de acceso por contraseña para manipular el prototipo modular, como se puede ver en la Figura 10. Una vez colocado nombre y contraseña correctamente, aparece un mensaje de bienvenida al operario en la vista frontal, cuando el usuario acepta el mensaje de bienvenida, automáticamente se visualizan los tres sistemas que integran el prototipo.

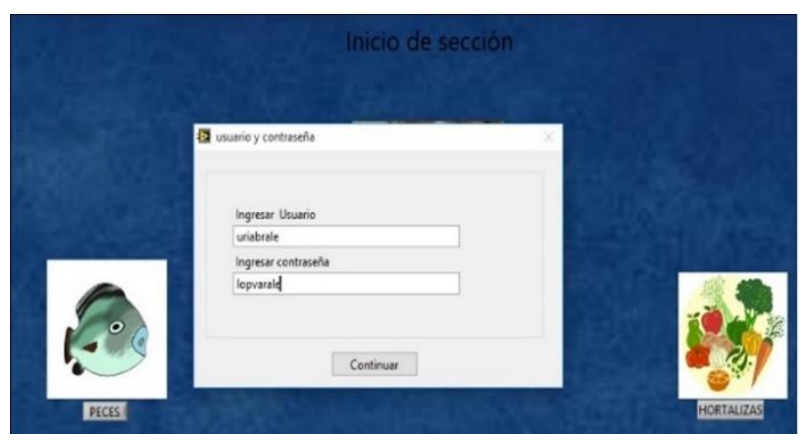

Figura 10 Sistema de acceso por contraseña Fuente: Elaboración propia

\section{Primer módulo: crianza de peces}

En la Tabla 4, se muestran varios muestreos de tres mediciones de $\mathrm{pH}$ en diferentes horarios para observar su variación, la primera medición fue del nivel de $\mathrm{pH}$ en el estanque sin la presencia de peces, la segunda se realizó una vez que el agua pasó por un filtro y la tercera prueba fue del estanque con la presencia de peces. Los resultados fueron los siguientes:

\begin{tabular}{|c|c|c|c|}
\hline Horario & \multicolumn{3}{c|}{ Nivel de pH } \\
& $\begin{array}{c}\text { Estanque } \\
\text { sin } \\
\text { presencia } \\
\text { de peces }\end{array}$ & $\begin{array}{c}\text { Después } \\
\text { del filtro }\end{array}$ & $\begin{array}{c}\text { Estanque } \\
\text { con } \\
\text { presencia } \\
\text { de peces }\end{array}$ \\
\hline 09:00 am & 7.91 & 7.72 & 7.00 \\
\hline $10: 00 \mathrm{am}$ & 7.98 & 7.00 & 7.12 \\
\hline $11: 00 \mathrm{am}$ & 7.91 & 7.38 & 7.07 \\
\hline $12: 00 \mathrm{am}$ & 7.93 & 7.38 & 7.15 \\
\hline $13: 00 \mathrm{am}$ & 7.89 & 7.12 & 7.20 \\
\hline $14: 00 \mathrm{pm}$ & 7.93 & 7.31 & 7.13 \\
\hline $15: 00 \mathrm{pm}$ & 7.91 & 7.26 & 7.12 \\
\hline $16: 00 \mathrm{pm}$ & 7.90 & 7.49 & 7.15 \\
\hline $17: 00 \mathrm{pm}$ & 7.85 & 7.28 & 7.21 \\
\hline $18: 00 \mathrm{pm}$ & 7.82 & 7.03 & 7.08 \\
\hline
\end{tabular}

Tabla 4 Comparación de los niveles de $\mathrm{pH}$ Fuente: Elaboración propia

\section{Segundo módulo: producción de hortalizas}

Se realizaron muestreos por tres días diferentes a un LDR en diferente horario y clima para observar la variación de luminosidad, véase en la Tabla 5, además se hizo un muestreo comparando dos LDR en un periodo corto, los resultados se presentan en la Tabla 6.

\begin{tabular}{|c|r|r|r|r|}
\hline Horario & \multicolumn{4}{c|}{ Unidad (Volts) } \\
& \multicolumn{1}{c}{ Día 1 } & \multicolumn{1}{c|}{ Día 2 } & \multicolumn{1}{c|}{ Día 3 } & Promedio \\
\hline 09:30 am & 3.72 & 3.06 & 3.64 & 3.47 \\
\hline 10:00 am & 4.00 & 3.18 & 3.90 & 3.69 \\
\hline 10:30 am & 4.38 & 3.92 & 3.93 & 4.07 \\
\hline 11:00 am & 4.38 & 4.60 & 3.98 & 4.32 \\
\hline 11:30 am & 4.12 & 4.26 & 4.00 & 4.12 \\
\hline 12:00 pm & 4.31 & 4.08 & 4.00 & 4.13 \\
\hline 12:30 pm & 4.26 & 4.81 & 4.10 & 4.39 \\
\hline 01:00 pm & 4.49 & 4.84 & 4.40 & 4.57 \\
\hline $01: 30 \mathrm{pm}$ & 4.28 & 4.88 & 4.80 & 4.65 \\
\hline $02: 00 \mathrm{pm}$ & 4.03 & 4.88 & 4.70 & 4.53 \\
\hline $02: 30 \mathrm{pm}$ & 3.72 & 4.88 & 4.90 & 4.50 \\
\hline $03: 00 \mathrm{pm}$ & 3.72 & 3.06 & 3.64 & 3.47 \\
\hline
\end{tabular}

Tabla 5 Comparación de los valores del LDR en tres días diferentes

Fuente: Elaboración propia

\begin{tabular}{|l|r|r|}
\hline Horario & \multicolumn{2}{c|}{ Unidad (Volts) } \\
& \multicolumn{1}{c}{ LDR 1 } & LDR 2 \\
\hline 07:30 pm & 0.103 & 0.165 \\
\hline 08:00 pm & 0.103 & 0.134 \\
\hline $08: 30 \mathrm{pm}$ & 0.103 & 0.134 \\
\hline 09:00 pm & 0.103 & 0.132 \\
\hline 09:30 pm & 0.103 & 0.130 \\
\hline 10:00 pm & 0.103 & 0.130 \\
\hline 10:30 pm & 0.103 & 0.130 \\
\hline $11: 00 \mathrm{pm}$ & 0.103 & 0.124 \\
\hline $11: 30 \mathrm{pm}$ & 0.103 & 0.124 \\
\hline $12: 00 \mathrm{am}$ & 0.103 & 0.124 \\
\hline
\end{tabular}

Tabla 6 Comparación de valores de dos LDR, en un día de máxima oscuridad

Fuente: Elaboración propia

CASTILLO-QUIROZ, Gregorio, CRUZ-GARRIDO, Arnulfo, ROJAS-BALBUENA, Dorian y LIMON-DIAZ, Miguel Ángel. Automatización y control de un prototipo modular autosustentable para la producción de alimentos. Revista de Ingeniería Industrial. 2020 
Con los datos anteriores se mantuvo un registro de datos sobre el tamaño promedio de la lechuga, el rábano y el cilantro con diversas circunstancias climatológicas, como se muestra en la Figura 11

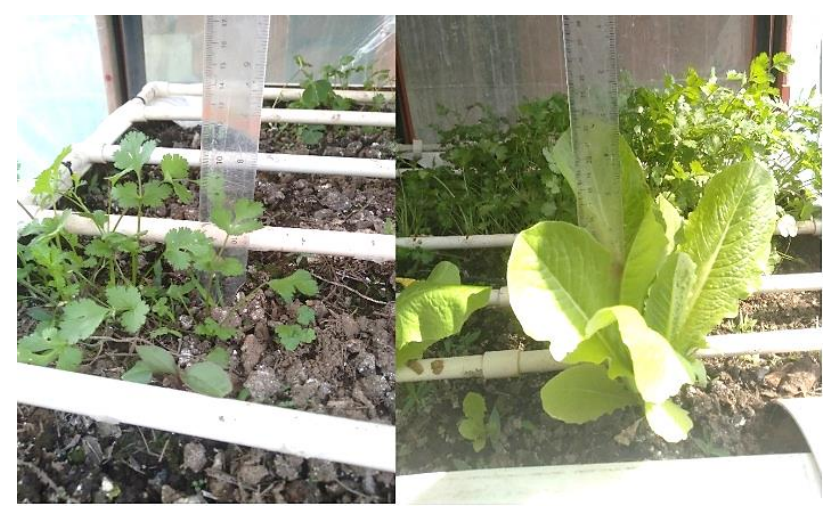

Figura 11 Registro de datos sobre el tamaño de los vegetales

Fuente: Elaboración propia

En la Tabla 7, se muestra una comparación en el crecimiento de las hortalizas, se manejaron dos cajones, un cajón (derecho) se le colocó una composta, se le dio riego abundante y con suficiente luz solar, mientras que al otro cajón (izquierdo) no se le colocó composta, el riego fue mínimo y la luz solar fue poca por la posición del módulo.

\begin{tabular}{|r|r|r|r|r|}
\hline Días & \multicolumn{2}{c}{$\begin{array}{c}\text { Unidad (cm), } \\
\text { cajón derecho }\end{array}$} & \multicolumn{2}{c|}{$\begin{array}{c}\text { Unidad (cm), } \\
\text { cajón izquierdo } \\
\text { Lechuga }\end{array}$} \\
\hline & 1.8 & 2.0 & 1.0 & 2.0 \\
\hline 10 & Cilantro & \multicolumn{1}{c|}{ Lechuga } & \multicolumn{1}{c|}{ Cilantro } \\
\hline 23 & 9.6 & 8.3 & 5.2 & 4.7 \\
\hline 46 & 17.3 & 15.0 & 11.1 & 7.8 \\
\hline 58 & 20.2 & 19.3 & 14 & 9.4 \\
\hline 64 & 22.5 & 21.5 & 16.5 & 11.0 \\
\hline
\end{tabular}

Tabla 7 Comparación de tamaños de hortalizas entre el cajón derecho e izquierdo

Fuente: Elaboración propia

Con lo anterior se concluyó que la composta que coloca antes del proceso de cultivación es mejor para la tierra debido a los nutrientes que esta le da, el riego no debe ser demasiado para que los rábanos no salgan aguañosos, pero tampoco puede ser mínimo por las lechugas y el cilantro.

Con las diferentes circunstancias que se pusieron en cada cajón, nos resultó que del lado derecho donde tenía buenas condiciones, se logró un $90 \%$ del $100 \%$ y del lado donde las circunstancias eran no óptimas, la producción fue de un $55 \%$ del $100 \%$.
Por lo cual, si colocamos una buena cantidad de composta generada por el tercer módulo, dependiendo del tipo de hortaliza, el riego sea constante y proporcionar la mayor cantidad de luz podríamos llegar a tener un $95 \%$ a $100 \%$ de producción.

\section{Tercer módulo: crianza de aves de corral}

El desarrollo de las aves con respecto al tiempo, podemos verlo el comportamiento de los datos que se muestra en la Tabla 8 , con la cual podemos determinar que su crecimiento fue el esperado, se logró mejorar la calidad de vida de las aves y esto trajo como consecuencia una menor mortalidad.

\begin{tabular}{r|r|}
$\begin{array}{c}\text { Tiempo } \\
\text { (Semanas) }\end{array}$ & $\begin{array}{c}\text { Peso } \\
\text { (gramos) }\end{array}$ \\
\hline 1 & 150 \\
\hline 2 & 350 \\
\hline 3 & 740 \\
\hline 4 & 1050 \\
\hline 5 & 1500 \\
\hline 6 & 2600 \\
\hline
\end{tabular}

Tabla 8 Desarrollo de las aves con respecto al tiempo Fuente: Elaboración propia

Es importante señalar que se observó una considerable disminución de gas amoniaco debido a la implementación de extractores y a la forma de extraer los desechos de las aves, con este resultado deducimos que se mejoró las condiciones del módulo, teniendo un mayor rendimiento en el crecimiento de las aves y una reducida contaminación, dado que los desechos de las aves se aprovechan como composta para hortalizas.

\section{Agradecimiento}

A la carrera de Ingeniería Mecatrónica del Tecnológico Nacional de México/Instituto Tecnológico Superior de Huauchinango por el apoyo y las facilidades en el desarrollo del proyecto, así mismo este artículo fue realizado como producto del proyecto "Diseño e integración de prototipo autosustentable vertical para crianza de peces, aves de corral y hortalizas, orientada a seguridad alimentaria en la Región de la Sierra Norte de Puebla" del cuerpo académico "Automatización y control de procesos", financiado por la convocatoria Fortalecimiento de Cuerpos Académicos del PRODEP. 


\section{Conclusiones}

El proyecto realizado contribuye aportaciones al sector alimentario en cualquier ambiente que se instale, en zonas rurales brinda al usuario de hortalizas y carnes frescas durante todo el año con un bajo costo de producción, en tanto en los sectores urbanos es una buena opción para disminuir el consumo de alimentos procesados y en ocasiones de dudosa procedencia.

El sistema implementado en el prototipo modular se controlaron las tres secciones, el diseño del sistema de monitoreo y control integrado fue diseñado para que cualquier persona pueda manipular e interpretar lo que sucede mientras el sistema está en funcionamiento, en caso de haber errores se podrán visualizar en forma de mensajes de advertencia o en forma de LED's indicadores en el panel de inicio del sistema.

Como resultado del proyecto, es posible concluir, que el sistema implementado nos proporciona una mejor alternativa en la producción de alimentos para autoconsumo.

\section{Referencias}

AGUILAR, N. E. S. (2020). Diseño de un sistema inteligente para el control automatizado de invernaderos.

CASTILLO, G., GÓMEZ, E., GONZAGA, E. y REYES, I. (2017). Sintonización y comparación de controladores para un aeropéndulo, Revista de Ingeniería Eléctrica, Vol. 1 No. 3, 16-26, http://www.ecorfan.org/republicofperu/research _journals/Revista_de_Ingenieria_Electrica/vol1 num3/ECORFAN_Revista_de_Ingenier\%C3\% ADa_El\%C3\%A9ctrica_VI_N3_3.pdf

Castillo G., Cruz, A., Gonzaga, E. y Luna, E. (2019). "Diseño e implementación de sistema de monitoreo automatizado en granja avícola", Revista de Investigación en Tecnologías de la Investigación (RITI), ISSN 2387-0893, 7 (14), Pp31-42.

doi:

https://doi.org/10.36825/RITI.07.14.011

Cruz-López, C. (2020). Sistema automatizado de control de variables fisicoquímicas en un prototipo para favorecer la apropiación social del cultivo de spirulina spp.
Durán, M. (2014). Criterios tecnológicoambientales bajo un enfoque sistémico: transferencia de tecnología química. Ingeniería Investigación y Tecnología, volumen XV (número 3), julio-septiembre, 339-350.

Fairchild Semiconductor Corporation (2014). "MOC3010M, MOC3011M, MOC3012M, MOC3020M, MOC3021M, MOC3022M, MOC3023M. 6-Pin DIP Random-Phase Optoisolators Triac Driver Output (250/400 volt peak)". En Fairchild Semiconductor Corporation. Consultado el 24 de octubre del $2019 . \quad$ Disponible en: http://www.farnell.com/datasheets/1806097.pdf

Genesis Gatica Porcayo, (2018). El desafío de la seguridad alimentaria en México. Consultado el 23 de octubre del 2019. Obtenido de Cienciamx: http://www.cienciamx.com/index.php/ciencia/a mbiente/24585-seguridad-alimentaria-mexico

INTAGRI. (2017). Acuaponia: Producción de Plantas y Peces. Recuperado el 08 de Septiembre de 2019, de: https://www.intagri.com/articulos/horticulturaprotegida/acuaponia-produccion-de-plantas-ypeces

Johana Andrea Ciro-Galeano, Mateo Itza-Ortiz. (2015). Parámetros Productivos. Recuperado el 08 de Septiembre de 2019, de Parámetros Productivos:

https://www.researchgate.net/publication/30835 6178_PARAMETROS_PRODUCTIVOS

Modificación de la Norma Oficial Mexicana NOM-127-SSA1-1994: Salud ambiental, agua para uso y consumo humano-Límites permisibles de calidad y tratamientos a que debe someterse el agua para su potabilización.

National Instruments (2015). "NI USB6008/6009". En National Instruments. Consultado el 22 de octubre del 2019. Disponible en: http://www.ni.com/pdf/manuals/371303n.pdf

Lidia Soria, (2018). El futuro de la alimentación se llama agricultura vertical. . Consultado el 20 de octubre del 2019. Obtenido de Compromiso Empresarial:

https://www.compromisoempresarial.com/rsc/2 018/08/el-futuro-de-la-alimentacion-se-llamaagricultura-vertical/

CASTILLO-QUIROZ, Gregorio, CRUZ-GARRIDO, Arnulfo, ROJAS-BALBUENA, Dorian y LIMON-DIAZ, Miguel Ángel. Automatización y control de un prototipo modular autosustentable para la producción de alimentos. Revista de Ingeniería Industrial. 2020 
Lopera, P. (2017). Medición de Parámetros Productivos en Avicultura. La revista global de avicultura, 23-26. Obtenido de Avicultura en producción ecológica:

https://avicultura.info/medicion-de-parametrosproductivos-en-avicultura/

López Camelo, A. F. (2003). Manual para la preparación y venta de frutas y hortalizas del campo al mercado. Organización De Las Naciones Unidas para la Agricultura y la Alimentación. ISBN 92-5-304991-X

http://www.fao.org/3/y4893s/y4893s06.htm

Páez Pazmiño, E. L. (2020). Diseño de un automatismo eléctrico de un proceso de Oxidación en una planta de tratamiento de aguas residuales para la industria cartonera.

Semiconductor Components Industries (2013). "P2N2222A". En Semiconductor Components Industries. Consultado el 23 de octubre del 2019. Disponible en: https://www.onsemi.com/pub/Collateral/P2N22 22A-D.PDF

Valderrama Gámez, A. S. Diseño e implementación de un sistema de control CNC compatible e Intercambiable para impresión 3d, corte y fresado de materiales blandos en aplicaciones didácticas.

Universitario, C. (2020). 0594-CU-P-2020 